\title{
Desarrollo de una Actividad de Aprendizaje Experiencial a través de un juego.
}

\author{
Ángel Peiró-Signes ${ }^{a}$, Oscar Trull-Domínguez ${ }^{b}$, Marival Segarra-Oña ${ }^{c}$, Blanca de \\ Miguel-Molina $^{\mathrm{d}}$
}

${ }^{a}$ Departamento de Organización de Empresas (Universitat Politècnica de València, anpeisig@omp.upv.es), bepartamento de Estadística e Investigación Operativa Aplicada y Calidad (Universitat Politècnica de València, otrull@eio.upv.es), 'Departamento de Organización de Empresas (Universitat Politècnica de València, maseo@omp.upv.es) y ${ }^{a}$ Departamento de Organización de Empresas (Universitat Politècnica de València, bedemigu@omp.upv.es),

\begin{abstract}
This paper focuses on the development of an Experimental Learning activity through a children's game. We want to show instructors a powerful tool to motivate and generate meaningful learning from a simple experience that allows us to test new teaching methodologies that are more appropriate to current educational environments. The activity is developed as a simulation of an industrial process using a car track game, where we intend to learn the basic principles to apply and analyze control charts. The results of the first two experiences indicate a high impact on the motivation and learning perception of the most basic concepts. On the other hand, the results also reveal the necessity for the instructor to emphasize the most complex concepts during the execution and the debriefing phases of the experience.
\end{abstract}

Keywords: Experiential Learning Activities, control charts, statistics, quality.

\section{Resumen}

Este artículo se centra en el desarrollo de una Actividad de Aprendizaje Experiencial a través de un juego de niños. Pretendemos mostrar a los instructores una potente herramienta de motivación y de generación de aprendizaje significativo a partir de una experiencia sencilla que nos puede permitir testar nuevas metodologías docentes más adecuadas a los entornos actuales de la educación. La actividad desarrollada a partir de la simulación de un proceso industrial mediante una pista de coches de juguete, donde pretendemos aprender los aspectos básicos para la aplicación y análisis de gráficos de control. El resultado de las primeras experiencias nos indican un alto impacto en la motivación y en la percepción de aprendizaje de los conceptos más básicos. Por otro lado, de los resultados también se desprende la necesidad del instructor de incidir en los conceptos más complejos de forma más importante durante las fases de ejecución y discusión de la experiencia.

Palabras clave: Aprendizaje experiencial, gráficos de control, estadística, calidad. 


\section{Introducción}

En el mundo educativo muchos aspectos han cambiado en los últimos años. El tiempo es escaso, las competencias que hay que desarrollar son muy amplias y mantener a los alumnos interesados en la materia cada vez es más y más duro.

En estas situaciones los profesores tenemos la opción de mantenernos con las metodologías que hemos ido usando durante años o enfrentarnos al cambio con decisión. El cambio supone en muchos casos adentrarnos en aspectos y ámbitos desconocidos de la enseñanza. Nuevas metodologías, nuevas actividades, una aproximación al problema diferente, etc. El cambio para adaptarse al nuevo entorno educacional parece cada vez más necesario.

Pensamos que la actividad en clase tiene que ser interesante para el alumno. Para despertar el interés en los alumnos tenemos múltiples opciones y cada una de las metodologías tiene sus elementos clave que permiten su aplicación con éxito. Además las actividades de clase deben estar relacionadas con el aprendizaje pasado (Ausubel,2000) y deben de poder ser recordadas por los alumnos después de algún tiempo. En otras palabras, las actividades deben producir un aprendizaje significativo y deben al mismo tiempo ser motivantes.

La experiencia nos indica que las Actividades de Aprendizaje Experiencial o Experiential Learning Activities (ELAs), son una herramienta poderosa para desarrollar aprendizajes experienciales sobre diversos conceptos, permiten trabajar competencias transversales y al mismo tiempo mantener el interés y la motivación en la asignatura (Peiró-Signes y SegarraOña, 2015), incluso en distintos niveles educativos (Peiró-Signes, 2015).

En este artículo se explica cómo se ha desarrollado una Actividad de Aprendizaje Experiencial (AAE) y los resultados preliminares de la experiencia. De esta forma, pretendemos mostrar a instructores o profesores un ejemplo de cómo a partir de un simple juego, podemos realizar un cambio en nuestra forma de abordar las clases para adaptarnos a este nuevo entorno educativo.

\section{Objetivos}

El cambio que tienen que realizar los instructores para introducir Actividades de Aprendizaje Experiencial en sus clases es grande. Hay un giro importante en cómo debe de enfrentarse el profesor a la sesión. En muchos casos requerirá que el propio profesor desarrolle nuevas habilidades y competencias.

El aspecto más importante a la hora de desarrollar una AAE es sin duda la planificación. Para planificar una AAE debemos considerar los conceptos que queremos transmitir, las habilidades y competencias que los estudiantes deben de tener y las que queremos desarrollar, entre otras. Además, debemos tener en cuenta aspectos relacionados con la infraestructura (tamaño y disposición de la clase, número de alumnos, recursos disponibles, etc.. El elemento clave en esta fase de planificación es determinar el propósito de la 
experiencia. Los objetivos de aprendizaje deben estar claros desde el primer momento para que toda la actividad pueda construir sobre ellos.

LaForge y Busing (1998) indicaron que el desarrollo de una AAE abarca desde la planificación hasta el debriefing o discusión final a través de las fases de introducción y ejecución de la actividad.

Este artículo se centra en el desarrollo de una AAE para mostrar a los estudiantes como construir, usar y analizar Gráficos de Control. Basándonos en un juego infantil hemos desarrollado una experiencia para cambiar la forma en que impartimos los conceptos relacionados con los gráficos de control. Con ello pretendemos conseguir un aprendizaje significativo y aumentar la motivación del estudiante durante la clase.

\section{Desarrollo de la innovación.}

\section{a. Planificación}

La planificación es vital para el desarrollo de la AAE. Es la fase clave para garantizar el éxito de la actividad. Empieza desarrollando los objetivos y continua con el desarrollo de las bases de la experiencia. Termina indicando cómo debe gestionarse y conducirse la fase final de debriefing.

Como indicaron Halpern y Hakelh (2003), desde el punto de vista del instructor la parte más complicada es el diseño de la experiencia. Una experiencia que sin la complejidad de una situación real, es capaz de reflejar de forma relevante los aspectos importantes que queremos transmitir. La experiencia debe permitir a los estudiantes tomar decisiones y reflexionar al final del proceso sobre aquellos aspectos que han ocurrido durante la experiencia (Kolb, 1984; Wheeler y McLeod, 2002). Es en esta fase final, es donde los estudiantes alcanzan el aprendizaje significativo.

La calidad ha sido un aspecto importante para las empresas en las últimas décadas. Dentro de la gestión de la calidad existen técnicas y metodologías que permiten recoger datos de un proceso, mostrar dichos datos y tomar decisiones a partir de su análisis. Los gráficos de control son una aplicación típica que permite monitorear procesos pasando por todos estos aspectos. Su aplicación permite detectar cuando están ocurriendo problemas en un proceso, diferenciar situaciones normales de anormales, establecer la estabilidad y los patrones del proceso o el resultado esperado. En otras palabras, permite diferenciar las causas comunes de variabilidad de las especiales.

La aplicación de los gráficos de control se divide en dos fases. En la primera se recogen los datos para establecer los límites de control y posteriormente se monitoriza el proceso para detectar causas especiales de variabilidad que nos indiquen que el proceso está fuera de control.

Los objetivos de aprendizaje en una sesión regular en esta área tienen que ver con la capacidad de aplicar y analizar gráficos de control: preparar y usar gráficos de control en un proceso, determinar situaciones fuera de control o diferenciar fuentes comunes de variabilidad. 
La experiencia ha sido diseñada sobre un juego de niños. El juego es bastante básico. Varios coches se mueven a través de una pista por la acción de la gravedad. Dos elevadores de correa, suben los coches de las zonas más bajas de la pista a las zonas más altas para que los coches se deslicen a través de una pista a modo de tobogán de nuevo hacia abajo. El juego tiene varios coches de colores distintos. Estos coches completan la vuelta en tiempos ligeramente distintos porque no son exactamente iguales y ni las trayectorias seguidas en la pista son exactamente iguales. Cada coche completa la pista, en promedio, en un tiempo por vuelta diferente, representando, cada uno de ellos un proceso distinto.

El objetivo de la experiencia es controlar el proceso (tiempo por vuelta) como un medio para lograr los objetivos mencionados anteriormente relacionados con los gráficos de control.

Para la experiencia necesitaremos otros recursos (ver fig. 1) además del juguete que se comentarán a continuación.

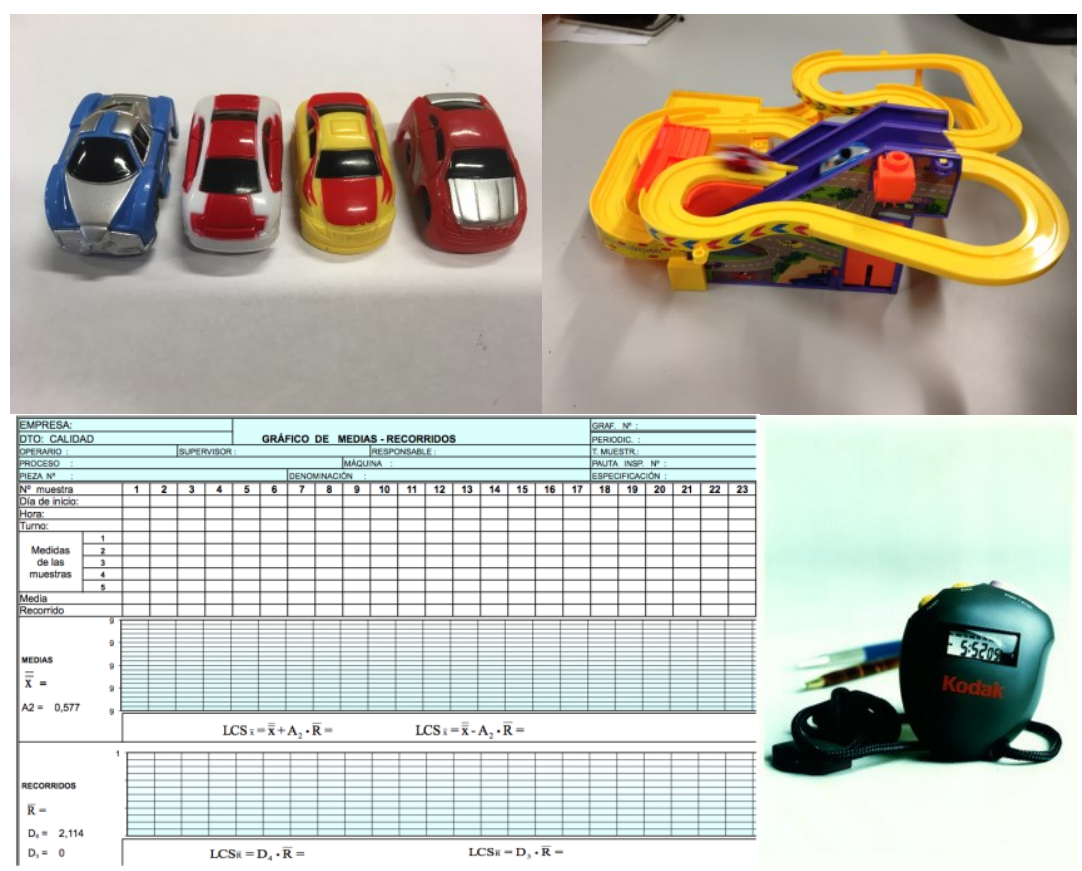

Fig. 1 Recursos de la actividad

\section{b. Introducción.}

Los materiales deben ser preparados por el instructor antes de que los estudiantes entren en el aula. El juego requiere partir el grupo en cuatro equipos de tres a cuatro estudiantes que serán asignados a cada coche. A cada estudiante se les asigna diferentes funciones: controlar el tiempo, anotar resultados, etc. 


\section{c. Actividad}

El primer objetivo es construir el gráfico de control. Necesitaremos una hoja de datos especial preparada por el profesor y que incluye los datos para la toma de muestras, cálculo de los límites de control y área milimetrada para el dibujo de los gráficos. En este caso, utilizamos un gráfico de control de rango promedio.

Los estudiantes necesitarán un cronómetro o teléfono móvil para controlar el tiempo por vuelta. Dos miembros de cada grupo comenzarán a tomar medidas de la vuelta en series de 5 vueltas. Inicialmente, los estudiantes permitirán que los coches funcionen de cuatro a seis vueltas hasta que el tiempo por vuelta sea más o menos estables y no se detecten problemas de circulación de los coches a lo largo de la pista. Escogiendo la parte superior de una de las cintas como el punto de inicio y final, los estudiantes harán 25 series de 5 medidas, dejando 3 a 4 vueltas entre series. Los resultados serán registrados por los otros miembros del grupo en la hoja de datos.

Una vez recuperados los datos "históricos", se instruirá a los estudiantes sobre el cálculo de la línea central y los límites de control superior e inferior, siguiendo las fórmulas indicadas en la hoja de datos.

El segundo objetivo es aprender a utilizar el gráfico de control. En este paso de la experiencia, el alumno tomará muestras a intervalos de muestreo especificados y trazarán valores en promedio y rango en una nueva hoja donde primero incluirán los límites de control previamente calculados.

Los límites de control son líneas de decisión, que en el primer análisis más simple permitirá a los estudiantes discriminar entre una proceso bajo control y uno fuera de control. Para esta parte, los roles de los estudiantes se cambian, para que todos los miembros puedan experimentar los diferentes roles dentro del equipo.

Después de unas cuantas rondas de medición, los estudiantes se darán cuenta de que los valores de promedio y rango trazados están entre los límites. El proceso es estable, funcionando dentro de los límites deseados. El proceso se está ejecutando bajo la influencia solo de causas comunes de la variabilidad y no deben adoptarse contramedidas.

En este momento se introducen causas especiales de variación. El juego está preparado para introducir varias causas de variación que podrían resultar en diferentes señales típicos de situaciones fuera control.

La primera señal de fuera de control es provocada deliberadamente por el instructor bajo la observación de los estudiantes. El instructor ralentiza las cintas poniendo el dedo sobre una de ellas mientras al menos uno de los coches se está elevando. Esto hará que uno o varios coches aumenten significativamente el tiempo de vuelta. Cuando los estudiantes trazan el resultado de la muestra el punto grafiado estará fuera del límite superior, lo que está indicando un comportamiento anormal del proceso. Esto puede alentar algunas discusiones sobre las causas de la variación que deberían introducir a los estudiantes en la siguiente parte de la experiencia. 
Para simular causas especiales, incorporamos un dispositivo de alimentación de corriente continua externa (DC). El juego original funciona con un paquete de 2 baterías del AA $1.5 \mathrm{~V}$ (fuente de alimentación $3 \mathrm{~V}$ ). Sin embargo, el motor de $\mathrm{CC}$ en el juguete es capaz de funcionar a un voltaje más alto y más bajo. Utilizamos una fuente de alimentación variable que nos permite alterar la tensión suministrada al juguete de 2 a $5 \mathrm{~V}$. Un aumento en el voltaje aumentará la velocidad del motor y viceversa. Aumentar la velocidad de la fuente de alimentación acelerará el proceso y eventualmente creará un resultado en un punto fuera de los límites de control. Preparamos un conjunto de experiencias para descubrir posibles situaciones fuera de control para que los estudiantes puedan experimentarlas.

La figura 2 muestra ejemplos de la configuración y el resultado esperado en el gráfico de control de control.
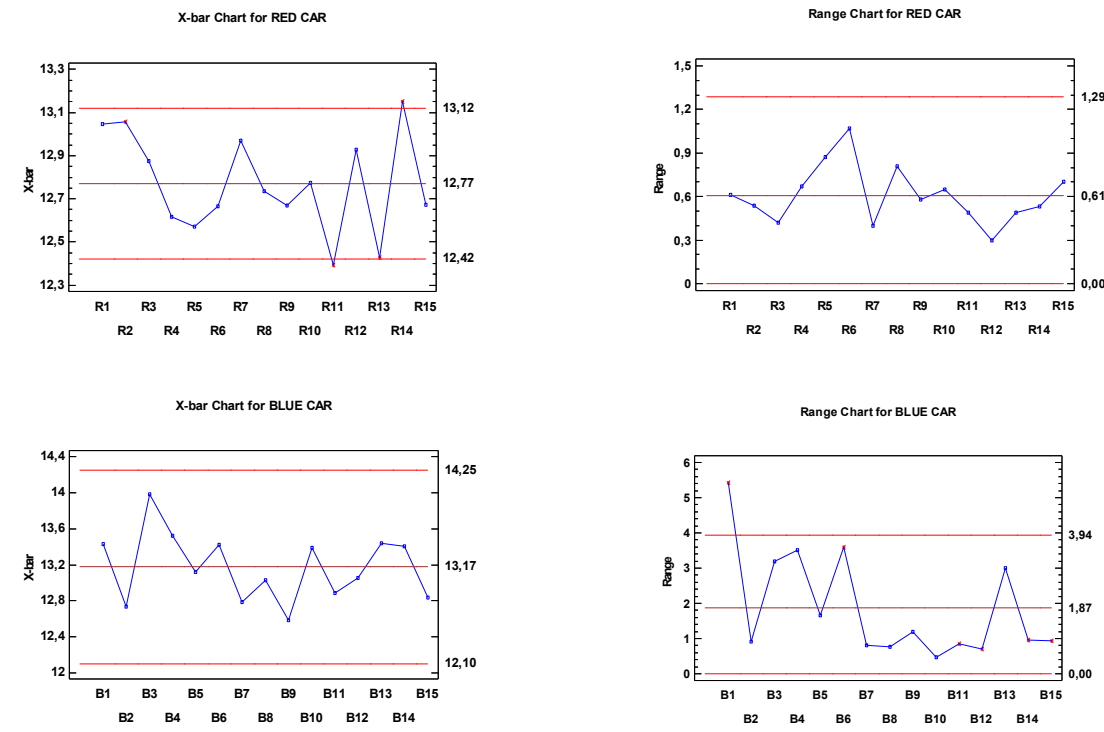

Fig. 2 Ejemplos de Gráficos de control obtenidos en la experiencia

Los estudiantes pasarán entre 30 minutos y 40 minutos para desarrollar todas estas experiencias.

\section{d. Debriefing}

Una vez completadas las experiencias los estudiantes discutirán durante 10 minutos los patrones que sigue cada una de las experiencia, las posibles razones de la variación en un proceso del mundo real.

La discusión comienza después de la discusión en pequeños grupos. El instructor resumirá la experiencia centrándose inicialmente en cuáles son las causas de la variabilidad común en el juego. Los estudiantes deben llegar con respuestas como: los coches no son exactamente los mismos (tienen diferentes formas, las ruedas y el eje no tienen la misma

(cc) EY-NC-ND 2017, Universitat Politècnica de València 
fricción, etc.), los coches no siguen exactamente el mismo camino (las curvas se toman de manera diferente, las cinta coge el coche de forma diferentemente cada vez, etc.), la cinta puede tener distinta velocidad (micro variaciones en la velocidad de la cinta), el controlador del tiempo no está parando el cronómetro en el mismo punto exacto cada vuelta, etc. Después de la primera parte de la discusión, las causas comunes deben estar claras y el instructor puede querer discutir las causas especiales y cómo afectan a la media y los valores de rango en el gráfico. El propósito es dejar que el estudiante se dé cuenta de que cuando el proceso está bajo variabilidad común todas las muestras estarán entre los límites y normalmente distribuidas alrededor de la línea central, mientras que las situaciones fuera de control terminarán en puntos fuera del límite o con patrones que están descubriendo comportamiento anormal.

El instructor debe usar situaciones reales relacionadas con el grado que los estudiantes están realizando para que visualicen su aplicación a un proceso real y asienten así los conocimientos. Por ejemplo, en un grado de ingeniería mecánica, podríamos usar el diámetro de un eje como ejemplo para mostrar situaciones que podrían ocurrir cuando el proceso de mecanización no está bajo control debido, por ejemplo, al desgaste de la herramienta, falta de lubricación en el proceso, error de posicionamiento de la herramienta o sobrecontrol del proceso.

Al final del periodo de retroalimentación y debate, el profesor puede reforzar los conceptos experimentados mediante una lección magistral, centrada en que los estudiantes evalúen de una forma más profunda sobre lo vivido en la experiencia para llegar a niveles superiores de aprendizaje (Meyers, 1986; Smith, 2003).

\section{Resultados}

Este artículo plantea la utilización de una actividad de aprendizaje experiencial en el aula. El éxito o fracaso de la actividad deberá evaluarse en forma de resultados. Para ello se ha previsto una evaluación de resultados después de introducir la actividad experiencial en base a las percepciones de los alumnos que la han realizado y su comparación con experiencias pasadas en la misma u otras asignaturas. Se plantea un pequeño cuestionario mediante una escala a Likert 1 a 5 donde 1 corresponde a totalmente en desacuerdo y 5 a totalmente de acuerdo.

Este test permite al instructor evaluar el impacto del ejercicio en particular y determinar los ajustes necesarios para conseguir los objetivos propuestos. La tabla 1 y la figura 3 muestran el resultado del cuestionario de evaluación de la actividad realizado sobre las dos primeras experiencias y un total de 32 alumnos. 
Tabla 1. Cuestionario y resultados de evaluación de la actividad

\begin{tabular}{|c|c|}
\hline Preguntas & Promedio \\
\hline $\begin{array}{l}\text { ¿Hasta qué punto se considera que la actividad ha ayudado al equipo a conocer } \\
\text { cómo se toman los datos necesarios para construir los Gráficos de Control? }\end{array}$ & 4,86 \\
\hline $\begin{array}{l}\text { ¿Hasta qué punto se considera que la actividad ha ayudado al equipo a conocer } \\
\text { cómo se calculan los límites de control? }\end{array}$ & 4,21 \\
\hline $\begin{array}{l}\text { ¿Hasta qué punto se considera que la actividad ha ayudado al equipo a conocer } \\
\text { como se monitoriza un proceso a través de los Gráficos de Control? }\end{array}$ & 4,64 \\
\hline $\begin{array}{l}\text { ¿Hasta qué punto se considera que la actividad ha ayudado al equipo a detectar } \\
\text { cuándo un proceso está bajo control utilizando Gráficos de Control? }\end{array}$ & 4,57 \\
\hline $\begin{array}{l}\text { ¿Hasta qué punto se considera que la actividad ha ayudado al equipo a detectar } \\
\text { cuándo un proceso está fuera de control utilizando Gráficos de Control? }\end{array}$ & 4,07 \\
\hline las posibles causas de que un proceso esté fuera control? & 3,79 \\
\hline $\begin{array}{l}\text { ¿Hasta qué punto se considera que la actividad ha ayudado al equipo a diferenciar } \\
\text { entre variabilidad común y variabilidad debido a causas especiales? }\end{array}$ & 4,50 \\
\hline $\begin{array}{l}\text { ¿En qué medida piensas que podrías realizar el control de un proceso por ti mismo } \\
\text { a partir de lo aprendido en la experiencia? }\end{array}$ & 3,93 \\
\hline $\begin{array}{l}\text { En qué medida ha sido motivadora la experiencia y preferible sobre una clase } \\
\text { magistral }\end{array}$ & 4,79 \\
\hline $\begin{array}{l}\text { ¿Crees que la actividad ha ayudado al equipo a tener la sensación de "en esto } \\
\text { estamos todos juntos"? }\end{array}$ & 4,71 \\
\hline ¿En que medida la comunicación ha sido importante para resolver la actividad? & 4,14 \\
\hline $\begin{array}{l}\text { ¿En que medida has podido participar y expresar tus opiniones en la discusión de } \\
\text { grupo y en la discusión general? }\end{array}$ & 4,07 \\
\hline $\begin{array}{l}\text { ¿Recomendarías la experiencia para el aprendizaje de los aspectos relacionados } \\
\text { con los Gráficos de Control? }\end{array}$ & 4,86 \\
\hline
\end{tabular}

Los resultados del test resaltan la potencia de la experiencia en relación a la motivación y a la adquisición de las habilidades y conceptos esenciales. Por otro lado, como era de esperar, los aspectos más complejos y de mayor nivel intelectual recibieron puntuaciones inferiores de los alumnos. Estos resultados preliminares nos indican los conceptos que deben reforzarse en futuras sesiones, especialmente en la parte de feedback y conclusiones, para garantizar un mayor cumplimiento de los objetivos planteados. 


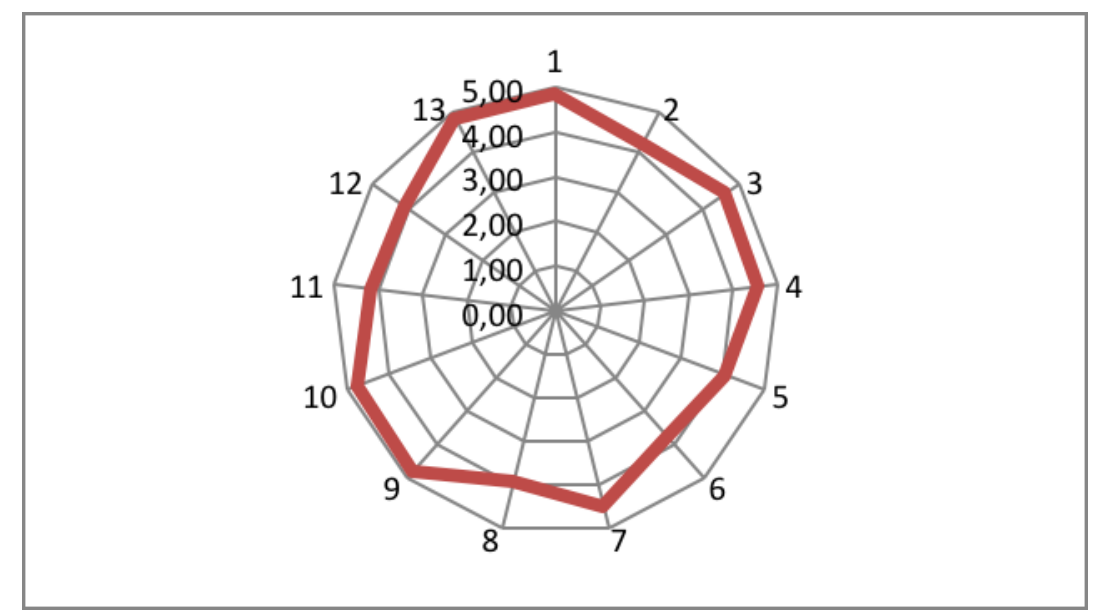

Fig. 3 Promedios de las preguntas de evaluación del uso de la AAE. Fuente: elaboración propia.

\section{Conclusiones}

Este artículo presenta el desarrollo de una actividad de aprendizaje experiencial diseñada para la enseñanza de los conceptos y habilidades necesarias en el uso y análisis de los Gráficos de Control. La actividad pretende ayudar a los alumnos dentro del proceso de enseñanza aprendizaje a conseguir un aprendizaje más significativo y relacionado con las actividades futuras de los egresados de asignaturas de grado en el área industrial y de ingeniería. Esta experiencia muestra cómo a partir del diseño de una Actividad de Aprendizaje Experiencial sencilla y mediante la utilización de un sencillo juego de niños se puede construir una sesión de aula motivadora para el alumnado y, que al mismo tiempo, permite a los alumnos visualizar aspectos complejos de los entornos productivos que, mediante metodologías docentes tradicionales, serían difíciles de cubrir.

Tras la experiencia acumulada se observa que el instructor debe poner especial énfasis en los conceptos y análisis de los aspectos más complejos de los Gráficos de Control en la fase de debriefing como refuerzo necesarios para alcanzar niveles significativos de aprendizaje en la experiencia.

Asimismo, es necesario evaluar si los resultados alcanzados son los esperados en las pruebas de evaluación de los conocimientos de la asignatura. El control de las percepciones de los alumnos a través del cuestionario y de los resultados a través de las pruebas de evaluación servirán al instructor para ajustar la experiencia y conseguir mayor eficacia en la consecución de los objetivos planteados.

\section{Agradecimientos}

Este trabajo forma parte del estudio desarrollado por el Grupo de Aprendizaje Experiencial (GAE) creado como EICE en la Universidad Politécnica de Valencia (UPV). Los autores desean agradecer a la UPV por el apoyo a través del PIME 2017 " Adaptación y desarrollo de aprendizajes experienciales al contexto de las asignaturas ". 


\section{Referencias}

HALPERN, D. y HAKEL, M. (2003). “Applying the science of learning”. Change, vol. 35, issue 4, p. $36-41$.

KOLB, D. (1984). Experiential learning: Experience as the source of learning and development.

Englewood Cliffs, NJ: Prentice Hall.

LAFORGE, R. y BUSING, M. (1998). "The use of industrial software to create experiential learning activities in operations management courses". Production and Operations Management, vol. 7, issue 3, p. 325-334.

MEYERS, C. (1986). Teaching students to think critically. San Francisco, CA: Jossey-Bass.

PEIRO-SIGNES, A., SEGARRA-OÑA, M., DE MIGUEL-MOLINA, M., J. ALBORS-GARRIGÓS, J. y DE-MIGUEL-MOLINA B. (2015). "The experiential learning activity: benefits and difficulties in real learning environments”. Proceedings of ICERI2015 Conference, p. 7231-7237.

PEIRO-SIGNES, A. y SEGARRA-OÑA, M.; (2015). "Experiential learning as a dynamizer of class activity”. Proceedings of ICERI2015 Conference, p. 1010-1013.

SMITH, G. (2003). "Beyond critical thinking and decision making: Teaching business students how to think". Journal of Management Education, vol. 27, issue 1, p. 24-51.

WHEELER, J., y MCLEOD, P. (2002). "Expanding our teaching effectiveness: Understanding our responses to in-the-moment classroom events". Journal of Management Education, vol. 26, issue 6, p. 693-716. 